\title{
Messungen von Bodenbewegungen auf West-Spitzbergen
}

Peter Fitze

Geographisches Institut Universität, Zürich

\section{Problemstellung}

Unsere Fließmessungen an einem Erdstrom im Schweizerischen Nationalpark (Furrer, Bachmann, Fitze; im Druck) ließen verschiedene Probleme offen, da wir diese Meßstelle nur periodisch mit einigen Wochen Abstand besuchen konnten. Im Nationalpark wurde in erster Linie festgestellt, wie groß die rezente Bodenbewegung ist, die Ursachen und der genaue Ablauf dieser Bewegung konnten dagegen nicht ermittelt werden. Spezielle Fragen, etwa die Einflüsse von Wassergehalt, Bodenzusammensetzung und auch der Hangneigung, blieben damals unbeantwortet.

Eine Möglichkeit, diesen Problemen näher auf den Grund zu gehen, bot sich bei der Spitzbergenexpedition des Geographischen Institutes der Universität Zürich im Sommer 1970. Es sei aber an dieser Stelle betont, daß ein fünfwöchiger Aufenthalt in Spitzbergen kaum genügt, diese Probleme mit aller
Gründlichkeit zu untersuchen. Dazu kam die gewichtsmäßige Beschränkung des in der Schweiz zu analysierenden Materials, da sich das Einrichten eines Feldlaboratoriums aus technischen Gründen leider als unmöglich erwies.

\section{Meßstellen und Meßmethoden}

Etwa $4 \mathrm{~km}$ westlich von Longyearbyen wurden auf einer $200 \mathrm{~m}$ hoch liegenden Hangverflachung in einem Strukturbodenfeld $5 \mathrm{Me}$ ßstellen eingerichtet. Alle Meßstellen befanden sich zwischen Steinstreifen; ihre Lage ist auf der Übersichtsaufnahme dargestellt (Meßstellen A bis E). Hangneigungsmessungen ergaben dabei folgende Werte:
A: $8^{\circ}$
D: $18^{\circ}$
B: $6^{\circ}$
E: $20^{\circ}$
C: $20^{\circ}$

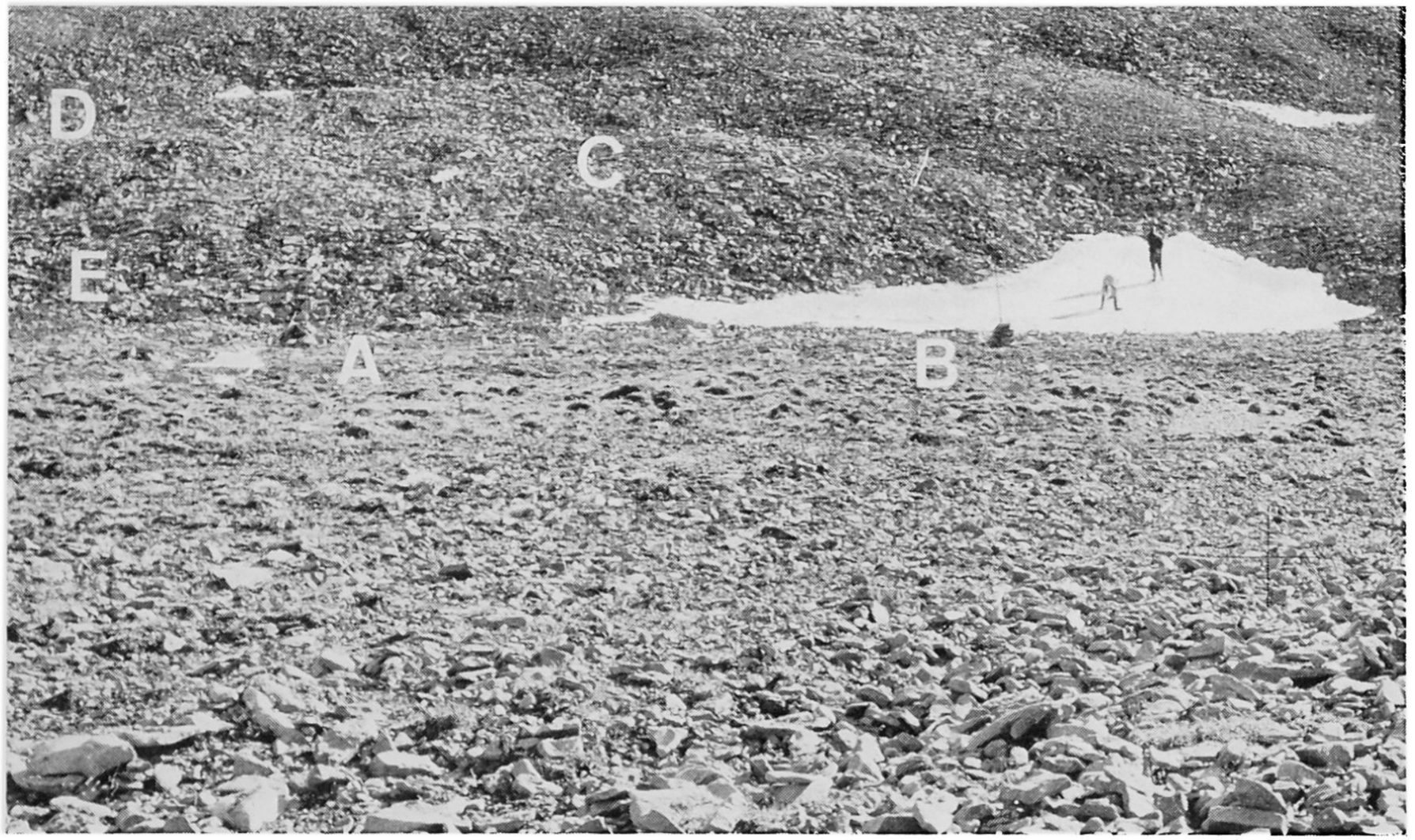

Abbildung 1. Ubersichtsaufnahme mit den fünf untersuchten Meßstellen (A bis E) 
Um Bodenbewegungen nachzuweisen, existieren verschiedene Methoden, wobei allerdings keine ganz befriedigend ist, vor allem was die Messungen in der Tiefe betrifft ${ }^{1}$. Da die Bodenbewegung an der Oberfläche am größten ist und mit zunehmender Tiefe rasch abnimmt (vgl. Rapp 1960, Smith 1960, Rudberg 1964, Furrer, Bachmann, Fitze, im Druck), beschränkten wir uns wegen der kurzen zur Verfügung stehenden Zeit nur auf Messungen der obersten Bodenschicht. Als Meßmarken dienten Nägel von 5, 10 und $15 \mathrm{~cm}$ Länge, die bis knapp zum Kopf in den Boden versenkt wurden. Um ein weiteres Versinken zu verhindern und zur besseren Erkennung wurden die Nagelknöpfe mit einem kleinen Styroporplättchen unterlegt. Der Grund für die Wahl von verschieden langen Nägeln liegt darin, ungleich starke Fließgeschwindigkeiten in verschiedenen Bodentiefen zu erkennen. Diese Methode, die sich im Nationalpark bewährt hatte, ließ hier allerdings keine unterschiedlichen Fließgeschwindigkeiten erkennen, vermutlich wegen der zu kurzen Beobachtungszeit. Die Aufnahmen der Meßstellen erfolgten jeweils mit Hilfe des Photoapparates.

Die absoluten Fließbeträge wurden mit Hilfe einer Basislinie aus Plastikseil gemessen, die wir in den Steinstreifen befestigten (ein Versuch, Eisenstangen im Permafrost zu verankern, scheiterte). Dieses Plastikseil schwebte nur wenige Zentimeter über den Meßstellen. Ein rechteckiger Holzrahmen diente als Stativauflage, er wurde bei jeder Aufnahme unter die Basislinie geschoben, womit wir folgende Zwecke errreichten:

Das Fotostativ konnte immer mit der gleichen Spreizung aufgestellt werden.

Die Aufnahmeebene blieb praktisch für alle Aufnahmen an einer Meßstelle konstant.

Der Rahmen konnte mit Hilfe von zwei darauf angebrachten Markierungen, die direkt unter die Basislinie zu liegen kamen, immer in die gleiche Position gebracht werden.

Neben diesen Bewegungsaufnahmen wurden von Zeit zu Zeit auch Bodenproben für Wassergehaltsbestimmungen entnommen, um eine eventuelle Abhängigkeit der Bodenbewegung vom Wassergehalt nachzuweisen. Wie Herz und Andreas (1966) zeigten, weicht der Wassergehalt bis in etwa $60 \mathrm{~cm}$ Tiefe nicht allzustark von jenem in $15 \mathrm{~cm}$ Tiefe ab, und viel tiefer reicht auch die Bodenbewegung nicht (vgl. Rapp 1960, Smith 1960, Elsasser 1968). Nur in den obersten $10 \mathrm{~cm}$ konnten Herz und Andreas ein sehr unterschiedliches Verhalten der Bodenfeuchte feststellen. Die an unseren Meßstellen entnommenen Bodenproben stammen aus einer Tiefe von 10 bis $15 \mathrm{~cm}$ und dürften demnach als repräsentativ für den sich bewegenden Teil des Bodens gelten. Die Entnahmedaten sind aus den Bewegungsdiagrammen ersichtlich.
Die Entnahme der Bodenprobe muß einerseits möglichst nahe der Meßstelle geschehen, aber andrerseits doch soweit davon entfernt sein, daß eine Beeinflussung der Fließgeschwindigkeit nicht anzunehmen ist, am besten oberhalb der Meßstelle. Die Proben wurden in Plastikfläschchen abgefüllt und mit Leim luftdicht verschlossen. Im Labor wurden später. Wassergehalt (durch Ofentrocknung bei $105^{\circ} \mathrm{C}$ ), Fließ- und Ausrollgrenze sowie die Korngrößenverteilung (durch Naßsiebung) bestimmt. Die Aufbewahrung der Proben in verleimten Plastikflaschen wurde vorher auf Wasserverlust getestet. Es zeigte sich, daß dieser Verlust in 10 Wochen etwa $1 \%$ des ursprünglichen Gehaltes betrug, ein Fehler, der auch bei der Probenentnahme oder der Verarbeitung im Labor auftreten kann.

\section{Bodenbewegung und ihre Ursachen}

Die Bewegungsdiagramme zeigen die Verhältnisse an den drei Meßstellen A, E und C. Aufgezeichnet sind die totalen Bewegungsbeträge, der Wassergehalt in Prozenten des Trockengewichtes in 10-15 cm Tiefe (Sterne), die mittlere Ausrollgrenze $\left(w_{\mathrm{a}}\right)$, die mittlere Fließgrenze $\left(w_{f}\right)$ und der Plastizitätsbereich (punktierte Fläche $z$ wischen $w_{a}$ und $w_{f}$ ). Für die Bestimmung dieser Grenzen sei auf Siedek und Voß (1966) verwiesen.

Die Bewegungsbeträge (schwarze Punkte) stellen das Mittel aus 12 Nägeln dar, je 4 von verschiedener Länge. Eine Bewegungsdifferenz zwischen kurzen und langen Nägeln konnte - wie bereits angedeutet worden ist - in dieser kurzen Zeit nicht festgestellt werden. Die Genauigkeit der ermittelten Bewegungsbeträge liegt nach den gemachten $\mathrm{Er}$ fahrungen bei etwa $2 \mathrm{~mm}$. Aus diesem Grund wurde die Meßstelle B nicht aufgezeichnet, da sie noch geringere Beträge als A aufweist ${ }^{2}$. D wurde in der Darstellung ebenfalls weggelassen, da die Charakteristik dieser Meßstelle sehr stark mit A und $\mathrm{E}$ vergleichbar ist.

Einen großen Unterschied zwischen den Meßstellen $A$ und $\mathrm{E}$ einerseits und $\mathrm{C}$ andrerseits zeigt die Fließgrenze und mit ihr auch der Plastizitätsbereich. Schon daraus läßt sich vermuten, daß der Boden in $\mathrm{C}$ anders zusammengesetzt ist, das heißt mehr "plastisches» Material (Silt und Ton) besitzt, was durch die Korngrößenanalyse bestätigt wird. Da sowohl $B$ eine ähnliche Bodenzusammensetzung wie $C$ als auch $\mathrm{D}$ eine ähnliche wie $\mathrm{A}$ und $\mathrm{E}$ besitzt, ist anzunehmen, daß zwischen den Meßstellen links in der Übersichtsaufnahme und jenen rechts eine Bodenänderung eintritt, die das Fließverhalten entscheidend beeinflußt. Diese Änderung beruht in erster Linie auf dem Tongehalt, der bei $A$ und $E$ ziemlich niedrig, bei $\mathrm{C}$ dagegen rund doppelt so gro $\beta$ ist. 

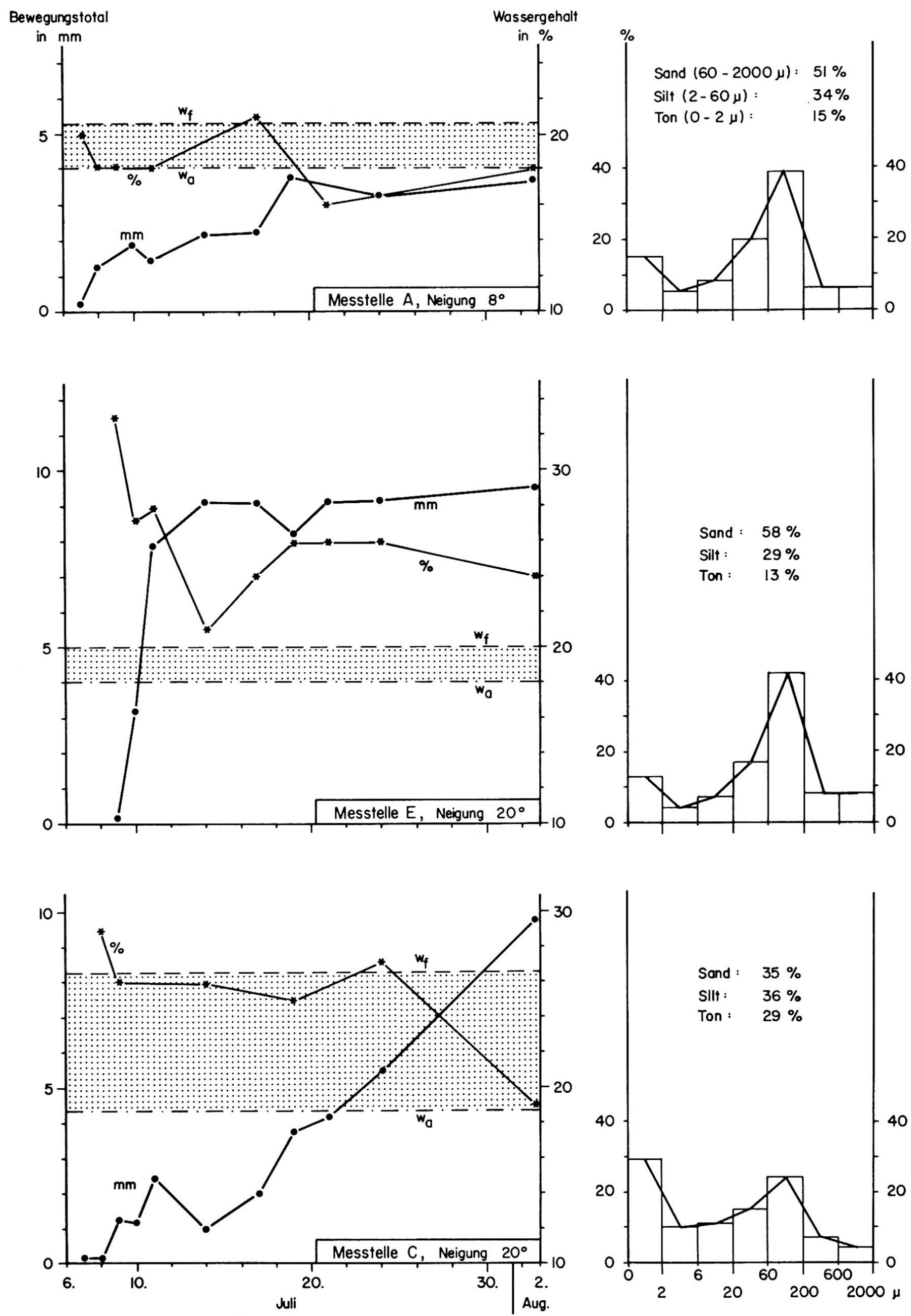

Figur 1. Bewegungsdiagramme (Erläuterung im Text) der drei Meßstellen A, E und $\mathrm{C}$ mit den zugehörigen Granulogrammen 
Umgekehrt verhält es sich mit dem Sandanteil, der bei $A$ und $E$ bedeutend höher als bei $C$ ist. Nach dem Vorschlag der Ämter für Bodenforschung (in: Scheffer-Schachtschabel, 1970) handelt es sich bei $A$ und $E$ um einen lehmigen Sand (IS) und bei $C$ um einen sandig-tonigen Lehm (stL).

Leider ist es sehr schwierig, einen direkten Einfluß der Materialzusammensetzung auf die Bodenbewegung nachzuweisen. Etwas eindeutiger gestaltet sich die Sache bei der Hangneigung und dem Wassergehalt.

Der Einfluß der Hangneigung scheint auf den ersten Blick deutlich hervorzutreten, denn der totale Fließbetrag liegt bei $\mathrm{A}$ (Hangneigung $8^{\circ}$ ) bei $4 \mathrm{~mm}$, bei $C$ und $E$ (Hangneigung $20^{\circ}$ ) bei $10 \mathrm{~mm}$. Doch dieser Totalbetrag setzt sich aus einzelnen Teilbeträgen zusammen, die stark gegen eine solche $\mathrm{Be}-$ hauptung sprechen. Im Zusammenhang mit dem Wassergehalt seien folgende Bemerkungen zu den Bewegungsdiagrammen gemacht:

Meßstelle A zeigt trotz niedrigen Wassergehaltes und geringer Hangneigung eine nachweisbare, schwache Bodenversetzung. Die Bewegung hört praktisch auf, wenn der Wassergehalt unter den der Ausrollgrenze sinkt (das Material befindet sich dann im halbfesten Zustand).

Bei E liegt der Wassergehalt durchwegs höher als bei $\mathrm{A}$, auch die Hangneigung ist größer. Trotzdem ist vom 14. Juli bis zum Ende der Beobachtungsperiode keine Bewegung mehr festzustellen. Der Wassergehalt liegt in diesem Zeitraum aber immer noch oberhalb der Fließgrenze. Eine Bewegung zeichnet sich bei dieser Meßstelle also erst dann ab, wenn der Wassergehalt mehr als $27 \%$ beträgt.

Bei C dagegen zeichnet sich eine fast kontinuierliche Bewegung ab, obwohl der Wassergehalt meist unterhalb der Fließgrenze liegt.

Der Vergleich zwischen $\mathrm{E}$ und $\mathrm{C}$ macht deutlich, daß für die Bewegung in erster Linie die Materialzusammensetzung verantwortlich ist, denn hier ergeben sich bei ähnlichem Wassergehalt und gleicher Hangneigung sehr verschiedene Beträge, wenn man die einzelnen Bewegungsphasen betrachtet.

Auch der Bewegungsunterschied zwischen A und E läßt sich keinesfalls mit der Hangneigung begründen, und der Wassergehalt scheint auch keine unmittelbare Rolle zu spielen. Der Grund ist auch hier in der Materialzusammensetzung zu suchen. Dieser Materialunterschied zwischen den beiden Meßstellen betrifft wohl nicht allein nur das unterschiedliche Verhältnis von Sand zu Silt und Ton. Der Anteil des Materials größer als $2 \mathrm{~mm}$, der nicht untersucht worden ist, spielt sicher auch eine wichtige Rolle. Darauf deutet jedenfalls die Beobachtung hin, daß das Einschlagen der Nägel bei E durch großen Steingehalt stärker behindert wurde als bei den übrigen Meßstellen.
Aufgrund dieser Messungen lassen sich drei Folgerungen ziehen:

Die Wirkung der Hangneigung darf nicht überschätzt werden. Eine Zunahme der Hangneigung ist nicht unbedingt gleichbedeutend mit einer $\mathrm{Zu}$ nahme der Bodenbewegung, wie häufig angenommen wird.

Der Wassergehalt zeigt einen ziemlich eindeutigen Stillstand der Bodenbewegung an, falls er einen tieferen Wert als den der Ausrollgrenze annimmt. Ein Wassergehaltswert aber, bei dem der Boden vom ruhenden in den bewegten Zustand übergeht, konnte als Verallgemeinerung nicht ermittelt werden (bei E dürfte dieser Wert um 27\% liegen). Ein Boden kann sich schon bewegen, wenn sich das Material noch im plastischen Zustand befindet (Meßstelle $C$ ) oder auch erst dann, wenn die Fließgrenze schon stark überschritten wird (Meßstelle E).

Der entscheidende Faktor ist die Materialzusammensetzung, wobei allerdings auch der Skelettanteil berücksichtigt werden sollte. Bei relativ niedrigem Wassergehalt dürfte steiniges und stark sandiges Material den Boden stabilisieren, lehmiges Material die Bodenbewegung fördern. Bei hohem Wasser gehalt scheinen sich die Verhältnisse umzukehren (vgl. E und C). Der steinige und sandige Boden bewegt sich fast schlagartig um relativ große Beträge, währenddem das lehmige Material auf eine Bewegung eher hindernd wirkt.

Diese Folgerungen zeigen deutlich, da $\beta$ eine allgemeine Aussage über das Fließverhalten verschiedener Böden kaum möglich ist wegen der von Fall zu Fall verschiedenen Verhältnisse. Auch den Vergleichen mit Fließbeträgen anderer Autoren (beispielsweise mit Dege 1943, Jahn 1960 und 1961) dürften aus diesen Gründen kaum allzuviel Bedeutung beigemessen werden. Bei künftigen Untersuchungen sollte also berücksichtigt werden, daß die granulometrischen Verhältnisse sowie die Zustandsgrenzen des Materials von entscheidender Bedeutung für die Bodenbewegung sind und allein schon wichtige Hinweise geben können. Mit dem tatsächliah gefundenen Wassergehalt zusammen sind Aussagen über die momentane Aktivität innerhalb gewisser Grenzen möglich. Eine längerfristige Prognose ist aber nur mit Hilfe vermehrter Wassergehaltsbestimmungen möglich, die uns über den $\mathrm{Zu}$ stand eines Bodens während längerer Zeit Aufschluß geben können.

\section{Zusammenfassung}

Die Messungen von Bodenbewegungen auf WestSpitzbergen während fünf Wochen erfolgten mit Hilfe von eingeschlagenen Nägeln, deren Stellungen photographisch festgehalten werden konnten. 
In gewissen Abständen wurden zusätzlich Bodenproben für Wassergehaltsbestimmungen entnommen. Aufgrund der Beobachtungen an drei Meßstellen konnten folgende Schlüsse gezogen werden: Die Bodenbewegung zeigt keine direkte Abhängigkeit von der Hangneigung.

Der Wassergehalt ist insofern von Bedeutung, als er das Aufhören der Bewegung anzeigt, wenn er die Ausrollgrenze unterschreitet. Ein allgemeinerGrenzwert für das Einsetzen der Bodenbewegung kannte nicht gefunden werden.

Entscheidend ist die Materialzusammensetzung. Bei nicht allzu hohen Wassergehaltswerten (bis etwa gegen $25 \%$ ) weist ein steiniger und sandiger Boden fast keine Bewegung auf, im Gegensatz zu lehmigem Material. Wichtig für zukünftige Untersuchungen sind auch die Anteile der Fraktionen größer als $2 \mathrm{~mm}$.

Aufgrund dieser Untersuchungen können Bodenbewegungen nicht verallgemeinert werden, es sind in jedem Fall die speziellen Verhältnisse zu berücksichtigen.

\section{Anmerkungen}

1 Für die verschiedenen Møthoden sei beispielsweise auf folgende Literatur verwiesen: Williams (1956), Rudberg (1958), Washburn (1960), Jahn (1961), Elsasser (1968), Zuber (1968).

2 Ein Vergleich lohnte sich auch darum nicht, weil der Wassergehalt bei B fast durchwegs unter dem der Ausrollgrenze lag, was bedeutet, daß das Material sich fast dauernd im halbfesten Zustand befand.

\section{Résumé}

A l'aide de mesurages de sols fluants à l'ouest du Spitzberg on a pu montrer que le mouvement de sol dépent premièrement de la composition du matériel. Les influences de la contenance d'eau et de la pente semblent être moins importantes.

\section{Literatur}

Dege W.: Über Ausmaß und Art der Bewegung arktischer Fließerde. Ztschr. f. Geomorph., Bd. XI, 1943.

Elsasser H.: Untersuchungen an Strukturböden im Kanton Graubünden. Diss. Univ. Zürich, 1968.

Furrer G., Bachmann F., Fitze P.: Erdströme im Schweizerischen Nationalpark. Im Druck.

Herz K., Andreas G.: Untersuchungen zur Ökologie der periglazialen Auftauschicht im Kongsfjordgebiet (West-Spitzbergen). Peterm. Mitt., Bd. 110, 1966.

Jahn, A.: Some remarks on evolution of slopes on Spitsbergen. Ztsch.r. f. Geomorph., Suppl. bd. 1, 1960.

- Polish I. G. Y. Spitsbergen expeditions in 1957, 1958, and 1959. Warszawa, 1961.

Rapp A.: Recent development of mountain slopes in Kärkevagge and surroundings, Northern Scandinavia. Geografiska Annaler, vol. XLII, Nr. 2-3, 1960.

Rudberg S.: Some observations concerning mass movement on slopes in Sweden. Meddelanden Fran Uppsala Universitets Geografiska Institution, Ser. A, No. 126, Bd. 1, 1958.

- Slow mass movement processes and slope development in the Norra Storfjäll area, Southern Swedish Lappland. Ztschr. f. Geomorph., Suppl.bd. 5, 1964.

Scheffer F., Schachtschabel P.: Lehrbuch der Bodenkunde. Ferd.-Enke-Verlag, Stuttgart, 1970.

Siedek P., Voß R.: Die Bodenprüfverfahren bei Straßenbauten. Werner-Verlag, Düsseldorf, 1966. Smith J.: Cryoturbation data from South Georgia. Biuletyn Peryglacjalny, Nr. 8, Lodz, 1960.

Washburn A. L.: Instrumentation for mass-wasting and patterned-ground studies in Northeast Greenland. Biuletyn Peryglacjalny, Nr. 8, Lodz, 1960.

Williams P. J.: Preliminary report of investigations into certain solifluction and patterned ground features in Norway. Cambridge, 1956.

Zuber E.: Pflanzensoziologische und ökologische Untersuchungen an Strukturrasen im Schweizerischen Nationalpark. Diss. Univ. Zürich, 1968. 